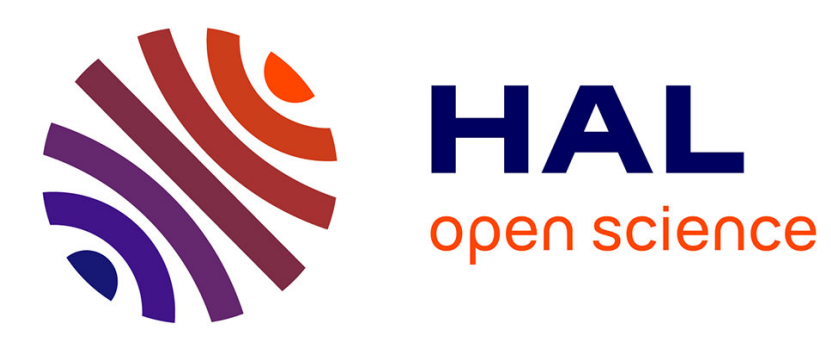

\title{
Agnès et ses soeurs: belles captives en enfance, de Molière à Baculard d'Arnaud
}

Christophe Martin

\section{To cite this version:}

Christophe Martin. Agnès et ses soeurs: belles captives en enfance, de Molière à Baculard d'Arnaud. Revue d'histoire littéraire de la France, 2004, 104, pp.343-362. 10.3917/rhlf.042.0343 . hal-01389902

\section{HAL Id: hal-01389902 https://hal.science/hal-01389902}

Submitted on 4 Nov 2016

HAL is a multi-disciplinary open access archive for the deposit and dissemination of scientific research documents, whether they are published or not. The documents may come from teaching and research institutions in France or abroad, or from public or private research centers.
L'archive ouverte pluridisciplinaire HAL, est destinée au dépôt et à la diffusion de documents scientifiques de niveau recherche, publiés ou non, émanant des établissements d'enseignement et de recherche français ou étrangers, des laboratoires publics ou privés. 


\section{Christophe Martin «Agnès et ses sours : belles captives en enfance, de Molière à Baculard d'Arnaud ", Revue d'Histoire Littéraire de la France, 2004, n 2, p. 323-342.}

Dans la littérature d'Ancien Régime, on le sait, l'enfant est, jusqu'à Rousseau, une figure pour le moins fugitive, qui ne fait que traverser la scène et reste, le plus souvent, à la lisière de la fiction ${ }^{1}$. Il est néanmoins un topos où, sous les espèces de la petite fille, la question de l'enfance occupe une place remarquable : celui de la "précaution inutile ", en particulier dans cette forme spécifique du motif qu'illustrent, au XVIIe siècle, Le Jaloux d'Estramadoure de Cervantès (dans les Nouvelles exemplaires, 1613), La Précaution inutile de Scarron (1656) et L'Ecole des femmes de Molière (première représentation en 1662) ${ }^{2}$. A superposer ces trois fictions classiques, c'est, en effet, une structure narrative analogue à bien des égards que l'on découvre, et où le caractère enfantin ou infantile de l'héroïne apparaît comme un enjeu majeur. Après avoir brièvement analysé les composantes de ce motif, c'est sur son devenir dans le roman du XVIIIe siècle qu'on voudrait s'attarder ici, en mettant en série trois fictions de " pédagogie négative » au siècle des Lumières.

Le scénario de la "précaution inutile» repose d'abord sur une motivation psychologique : la jalousie obsessionnelle d'un bourgeois vieillissant et enrichi, prêt à tout pour ne point être la dupe des femmes et de leurs "subtiles trames" pour paraphraser Arnolphe. ${ }^{3}$ Obsession du cocuage et hantise de la duplicité féminine que ces barbons héritent peut-être du Pygmalion d'Ovide, "révolté des vices dont la nature a rempli le cœur des femmes $»^{4}$, et qui sera celle encore du Roi dans la Grisélidis de Perrault, en 1695 : à l'instar de ce dernier, tous se définissent, d'un point de vue caractérologique, par un tempérament « chagrin et mélancolique » qui leur fait voir « tout le beau sexe infidèle et trompeur ${ }^{5}$. On sait qu'après un long célibat qui désespère sa cour, le Roi de la nouvelle de Perrault épouse une très jeune bergère en laquelle il a découvert avec enchantement « une simplicité, / Une douceur, une sincérité, / Dont il croyait le beau sexe incapable $»^{6}$. Jeune et non point petite fille, donc, que cette ravissante Grisélidis. On soulignera néanmoins que le regard du prince est d'abord séduit par une bouche qui « de l'enfance avait tout l'agrément »... De même, Carrizalès chez Cervantès, Dom Pedre chez Scarron et Arnolphe chez Molière finissent tous par élire une enfant, ou une très jeune femme explicitement rattachée à l'enfance, sans bien ni ressource : dans Le Jaloux d'Estramadoure, Léonore a certes treize ou quatorze ans lorsque Carrizalès l'épouse mais ce dernier estime que « ce n'est encore qu'une enfant ${ }^{7}$; dans $L a$ Précaution inutile, Laure est recueillie dès la naissance par Dom Pedre qui l'a met au couvent à l'âge de trois ans en attendant de pouvoir l'épouser. Et l'on sait, qu'Agnès a quatre ans lorsqu'Arnolphe en tombe amoureux :

\footnotetext{
${ }^{1}$ Voir à ce sujet le numéro spécial de la revue Littératures classiques intitulé «Enfance et littérature au XVIIe siècle », coordonné par Andrée Mansau (1991, $\left.\mathrm{n}^{\circ} 14\right)$.

2 La nouvelle de Scarron, traduite du récit de Maria de Zayas y Sotomayor, El Prevenido engañado (1637) est, on le sait, la principale source d'inspiration de Molière pour L'Ecole des femmes. Pour une étude comparée des deux textes, voir Nathalie Fournier, « De La Précaution inutile à L'Ecole des femmes: la réécriture de Scarron par Molière », XVIIe siècle n ${ }^{\circ} 186,1995$, p. 49-60.

3 « Je sais les tours rusés, et les subtiles trames, / Dont pour nous planter, savent user les femmes, / Et comme on est dupé par leurs dextérités » (L'Ecole des femmes, I, sc.1, v. 75-76).

4 Ovide, Les Métamorphoses, X, 243 (trad. G. Lafaye, Paris, Gallimard, 1992, p. 329).

5 Perrault, Grisélidis, in Contes, éd. J.-P. Collinet, Paris, Gallimard, 1981, p. 60.

6 Ibid., p. 65.

${ }^{7}$ Cervantès, Le Jaloux d'Estramadoure, in Nouvelles exemplaires, trad. J. Cassou, Paris, Gallimard, 1949, p. 310 .
} 
Un air doux et posé, parmi d'autres enfants,

M'inspira de l'amour pour elle dès quatre ans. ${ }^{8}$

Choix d'objet éloquent et qui s'explique aisément : comme dit Carrizalès, « la tendresse [des] ans » de l'objet élu (ainsi, bien entendu, qu'une infériorité prodigieuse - du moins en apparence en ce qui concerne Agnès - de rang ou de fortune) permet de « rassurer les soupçons » du jaloux. ${ }^{9}$ Cette extrême jeunesse n'est pas seulement gage d'innocence : elle comble surtout le fantasme d'une emprise absolue sur l'objet du désir. Car contrairement à l'heureuse découverte de Grisélidis par le prince chez Perrault, l'élection de ces petites filles ne relève ni du hasard, ni du merveilleux. Elle n'est que l'étape initiale d'une stratégie parfaitement concertée et patiemment mise en œuvre, devant conduire à une complète appropriation. Nulle rencontre miraculeuse ici : chacune des petites filles choisies fait l'objet d'une pure et simple acquisition. Carrizalès négocie la main de Léonore auprès de ses parents, Dom Pedre recueille Laure abandonnée par sa mère et Arnolphe achète Agnès à une mère « se trouvant de pauvreté pressée » (v. 131).

Une fois l'objet précieux en leur pouvoir, chacun n'a de cesse de s'en assurer une jouissance exclusive. D'où la troisième composante du scénario:l'isolement et la séquestration de la femme-enfant en un lieu où le tyran domestique dissimule son trésor. ${ }^{10}$ Dans le cas de Laure et d'Agnès, la claustration est d'abord conventuelle, phase intermédiaire que la bienséance rend nécessaire étant donné l'âge des deux petites filles. A peine sorties de l'enfance, elles ne tardent pas, toutefois, à connaître un sort analogue à celui de Léonore, recluse dans une maison visiblement conçue sur le modèle du cloître : non content d'avoir fait surélever les murs qui donnent sur la rue, Carrizalès a placé un tour entre le vestibule et le patio et choisi pour domestiques des femmes exclusivement, de manière à préserver sa demeure de toute intrusion masculine. On sait aussi combien il importe à Arnolphe de soustraire le corps d'Agnès à tout commerce avec le monde :

Je l'ai donc retirée ; et comme ma demeure

A cent sortes de monde est ouverte à toute heure

Je l'ai mise à l'écart, comme il faut tout prévoir

Dans cette autre maison, où nul ne me vient voir (I, sc.1, v. 143-146)

Chez Cervantès comme chez Molière, le fantasme d'exclusivité s'étaye sur un dispositif spatial complexe, entièrement conçu pour la claustration de la femme-enfant.

Cette clôture physique est redoublée d'une clôture pour ainsi dire mentale. Une autre composante fondamentale du topos est, en effet, la dimension essentiellement «négative » (pour reprendre, en en déplaçant légèrement la valeur, une notion chère à Rousseau) de l'éducation reçue par la femme-enfant. Il s'agit de maintenir ces belles captives dans une bienheureuse " innocence » en vertu d'une pédagogie paradoxale qui ne vise rien d'autre qu'une rigoureuse infantilisation. Carrizalès gave Léonore de sucreries et la laisse jouer à la poupée. ${ }^{11}$ Dom Pedre donne ordre que Laure " n'eût aucune connaissance des choses du

\footnotetext{
8 L'Ecole des femmes, I, sc. 1, v. 129-130.

${ }^{9}$ Le Jaloux d'Estramadoure, p. 310.

10 Même si cela ne fait pas partie, en l'occurrence, d'un projet concerté, on soulignera que la Grisélidis de Perrault subit très vite une réclusion analogue. Son époux ne tarde pas, en effet, à retomber dans son humeur jalouse : «Dans son palais il la tient resserrée, / Loin de tous les plaisirs qui naissent à la Cour, / Et dans sa chambre, où seule elle vit retirée, / A peine il laisse entrer le jour » (p. 75).

11 «La simplicité [de Léonore] l'achemina même à jouer à la poupée et à tels autres enfantillages qui découvraient son jeune âge et la candeur de son naturel. Tout cela était propre à satisfaire grandement le mari jaloux » (Le Jaloux d'Estramadoure, p. 314).
} 
monde ». ${ }^{12}$ Et Arnolphe, craignant de gâter « l'heureux naturel » d'Agnès, ne se soucie que de la rendre « idiote autant qu'il se pourrait» $(\mathrm{I}, \mathrm{sc} .1, \mathrm{v} .138)^{13}$.

Mais la donnée la plus essentielle de ce topos est en réalité indiquée dès le titre de la nouvelle de Scarron: c'est bien l'inutilité de la "précaution" prise par ces barbons qui détermine, en effet, la structure narrative de ces trois fictions. ${ }^{14}$ Par définition générique, le projet des jaloux est voué à l'échec. Telle est la source comique d'un canevas dont la nature farcesque est évidente. Dans tous les cas, l'intrigue se construit autour de la ruine d'un idéal de maîtrise totalement chimérique et vain. Entreprises ridicules ou dérisoires, où l'énergie prodigieuse déployée par ces vieux barbons offre un contraste saisissant avec la nullité du résultat obtenu. Car la forteresse n'est évidemment pas imprenable et chacune de ces jeunes captives ne tarde pas à trouver son Persée en de séduisants jeunes hommes qui parviennent à déjouer les vaines précautions des barbons, et à faire sortir leur Andromède de leur cage et de leur hibernation. ${ }^{15}$ Plus fondamentalement, l'échec est inscrit dans la nature même de ces petites filles, nature sur laquelle les barbons jaloux ont finalement échoué à exercer leur emprise. Ainsi, le projet de réification et de dénaturation conçu par Arnolphe ne saurait empêcher la manifestation de la "vraie » nature d'Agnès, ni l'éveil rapide de sa conscience longtemps engourdie. ${ }^{16}$ La petite captive, objet d'abord dénué de parole propre (véritablement infans), accède peu à peu au statut de sujet parlant. ${ }^{17} \mathrm{C}$ 'est à une seconde naissance de la petite fille, jusqu'alors maintenue dans une éternelle enfance mais désormais éclairée par l'amour, que conduit le scénario de la précaution inutile. ${ }^{18}$

Même s'il s'agit d'une filiation inaperçue, semble-t-il, ce motif n'est pas sans postérité au siècle des Lumières. On retiendra ici trois récits : 1'histoire de Céladine dans Les Délices $d u$ sentiment du chevalier de Mouhy (1753), l'histoire de Tiamy dans les Mémoires de deux amis de H.-F de La Solle (1754) et Liebman de Baculard d'Arnaud (1775). Rien de commun dans le registre ni la tonalité de ces trois textes : le romanesque débridé et fort peu soucieux de vraisemblance propre à Mouhy contraste aussi bien avec la froideur presque clinique du récit de La Solle qu'avec le registre « sensible » de la nouvelle de Baculard d'Arnaud. ${ }^{19}$ Mais

12 Scarron, La Précaution inutile, in Nouvelles tragi-comiques, éd. Roger Guichemerre, Paris, Nizet, 1986, p. 41.

13 Sur l'importance de cette «pédagogie négative » dans la comédie de Molière, voir notamment R. Albanese Jr., " Pédagogie et didactisme dans L'Ecole des femmes », Romance notes, vol. XVI, n 1, 1974, p. 114-123 et Barbara Johnson, « Teaching ignorance : L’Ecole des femmes », Yale French Studies, n 63, 1982, p. 165-182.

14 Ce sera encore, on le sait, le sous-titre du Barbier de Séville de Beaumarchais en 1775.

15 Rôle tenu par Loayasa dans le Jaloux d'Estramadoure, par un gentilhomme de Cordoue dans La Précaution inutile, par Horace dans L'École des femmes. Citons pour leur caractère exemplaire ces quelques lignes de la nouvelle de Cervantès : «Léonore se rendit, Léonore se trahit, Léonore se perdit, jetant à terre toutes les belles précautions du sagace Carrizalès » (Le Jaloux d'Estramadoure, p. 341).

16 Agnés passe « de l'animalité obtuse à l'ingénuité charmante puis à la transparence indécise pour émerger à la conscience, à la souffrance et à la poésie » (Patrick Dandrey, préface à L'Ecole des femmes, Paris, LGF, Le Livre de Poche, 2000, p. 30).

17 Voir Bernard Magne, "L'Ecole des femmes ou la conquête de la parole », Revue des Sciences Humaines $\mathrm{n}^{\circ}$ 145,1972 , p. $125-40$.

18 La complexité et l'ironie du dénouement de la nouvelle de Cervantès appelleraient des développements détaillés qui n'ont pas leur place ici. On rappellera seulement la leçon que dégage le narrateur à la fin de son récit sur le «peu de fiance qu'il faut mettre en clefs, tours et murailles, alors que la volonté demeure libre » (Le Jaloux d'Estramadoure, p. 350).

19 On ne saurait déterminer avec certitude le jeu des influences réciproques entre ces trois récits. Dans la nouvelle de Baculard d'Arnaud, une note de l'auteur suggère un lien possible avec l'un des deux textes antérieurs : «j'ai lu dans mon extrême jeunesse, un petit ouvrage, dont je ne saurais me rappeler le titre, et qui a, je crois, quelque conformité avec le fonds de cette anecdote » (Liebman. Histoire allemande, éd. H. Coulet, in Pygmalions des Lumières, Paris, Desjonquères, 1998, p. 124. Le texte retenu est l'édition de 1789, révisée par 
ce qui frappe d'emblée, dans ces différentes reprises du scénario de la belle captive en enfance, c'est la transformation radicale des amants geôliers : si leurs stratagèmes sont bien analogues à ceux de Carrizalès ou d'Arnolphe, leur portrait et à certains égards aussi leurs motivations sont fort différents.

Le puissant prince Cornandouk, dans Les Délices $d u$ sentiment, n'a rien d'un personnage ridicule : dégoûté du pouvoir par les intrigues et la tyrannie de son frère, il décide de renoncer à toute ambition royale pour passer le reste de ses jours dans une retraite solitaire. Sa défiance envers les femmes l'apparente plutôt au Roi dans Grisélidis qu'au jaloux moliéresque (avant d'enlever la petite Céladine, il s'éprend d'ailleurs comme lui d'une jeune paysanne, âgée de dix-sept ans ; mais en dépit de son air naïf et sincère, celle-ci trahit sa confiance). L'humeur mélancolique du Liebman de Baculard d'Arnaud n'a rien de comique non plus : loin de relever d'une clinique classique des humeurs, son âme tourmentée en fait plutôt un cousin de Werther, son quasi contemporain, ${ }^{20}$ et permet à Baculard de situer son récit dans la tonalité hautement pathétique qui lui est habituelle. Par son âge mûr et son statut de spéculateur prodigieusement enrichi dans le système de Law, le chevalier de Borille, chez La Solle, est sans doute l'héritier le plus direct des barbons du XVIIe siècle. Au reste, son projet de séquestrer une jeune beauté « sur la fidélité de laquelle il pourrait compter, sans être aussi fou que les autres hommes, qui ne pouvaient jamais, selon lui, être sûrs du cœur d'une femme » est très proche de celui d'Arnolphe qui déclarait : " épouser une sotte est pour n'être point sot " (v. 82). ${ }^{21}$ Mais la jalousie n'est pas la caractéristique essentielle du personnage (le mot n'est même d'ailleurs jamais prononcé), qui se définit avant tout par sa propension à se «livre[r] totalement à sa spéculation malade de la peur de mourir » (MDA, t. 3 , p. 131). Au lecteur qui se demanderait en quoi cette angoisse de la mort peut expliquer le projet de séquestrer une petite fille pour en faire un objet de plaisir, le récit de La Solle ne fournit aucune explication. ${ }^{22}$ Tout se passe comme si la caractérologie classique était devenue impuissante à rendre compte de l'extravagance du personnage. Loin d'être réductible à une typologie traditionnelle, le rapport de Borille au féminin est placé tout entier sous le signe de l'ambivalence : «il aimait les femmes qu'il méprisait, et désirait avec ardeur les plaisirs que ce mépris lui empêchait de se procurer » $(M D A$, t. 3, p. 131). C'est une même ambivalence qui caractérise en profondeur les relations de Cornandouk avec Céladine et de Liebman avec Amélie. Le terme de jalousie ne suffit d'ailleurs pas davantage à définir l'obsession de ce dernier : sa motivation essentielle n'est plus une défiance envers la vertu féminine mais le

l'auteur. Nous abrégeons désormais en Liebman). Selon Servais Etienne, il s'agirait du récit de La Solle (voir Le Genre romanesque en France depuis l'apparition de 'La Nouvelle Héloïse' jusqu'aux approches de la Révolution, Bruxelles, 1922, p. 76). Mais d'après Robert Dawson, qui n'écarte pas cette première hypothèse, il s'agirait plutôt de Pigmalion ou la statue animée de Boureau-Deslandes, publié en 1741 (voir R. Dawson, Baculard d'Arnaud: life and prose fiction, Oxford, Voltaire Foundation, 1976, SVEC 141-142, t. 1, p. 410). En réalité, il nous semble que le texte avec lequel la nouvelle de Baculard d'Arnaud entretient les rapports les plus étroits est le récit enchâssé dans Les Délices du sentiment de Mouhy, titre dont on peut percevoir un écho, d'ailleurs, dans celui du recueil dont Liebman fait partie : Les Epreuves du sentiment.

20 Voir à ce sujet R. Dawson, op. cit., t. 1, p. 404-444..

${ }^{21}$ H.-F. de La Solle, Mémoires de deux amis ou Les Aventures de Barniwal et Rinville (Londres, 1754), t. 3, p. 147 (désormais $M D A$ ). Dans sa préface, La Solle souligne d'ailleurs, non sans provocation, la banalité du projet de son personnage : «Bien des hommes ont à peu près les mêmes projets que lui parce qu'ils ont de la fidélité des femmes la même opinion. Les seules difficultés leur font abandonner le dessein » (t. 1,p. IX). Pour un résumé détaillé de cet épisode, voir Jacques Rustin, «Une "expérimentation” libertine dans le roman du XVIIIe siècle : l'épisode de Tiamy dans les Mémoires de deux amis (De La Solle, 1754)», Travaux de linguistique et de littérature, IX (2), 1971, p. 85-96.

22 Nous renvoyons sur ce point à une précédente étude : «Belle captive en enfance. Dérives d'un topos littéraire dans les Mémoires de deux amis de Henri-François de La Solle », in Folies romanesques au siècle des Lumières. Actes du colloque de la Sorbonne Nouvelle (11-13 décembre 1997), éd. R. Démoris et H. Lafon, Paris, Desjonquères, 1998, p. 365-379. 
désir narcissique de trouver " une âme qui réponde à [son] âme », un « ami d'un autre sexe » (Liebman, p. 121 et p. 123), autrement dit une amante en laquelle il puisse voir se refléter la perfection de son être et de sa sensibilité.

Mais ce qui distingue plus radicalement encore ces trois récits des fictions du XVIIe siècle dont ils reprennent la donnée de base, c'est la mise en évidence du désir proprement pédophile dont Céladine, Tiamy et Amélie font l'objet. Assurément, le spectre de la pédophilie planait déjà de manière assez insistante chez Cervantès, et plus encore chez Molière. ${ }^{23}$ Car maintenir Agnès en enfance, c'est sans doute avant tout, pour Arnolphe, le moyen d'éterniser la première vision de cette enfant de quatre ans, dont l' " air doux et posé » (v. 129) et les « attraits naissants » (v. 1030) ont provoqué en lui un authentique émoi. Mais, bienséances obligent, la parenthèse du couvent écartait toute idée d'un contact prolongé entre le barbon et la petite fille. Dans le scénario de la précaution inutile, celle-ci n'est pas censée être en elle-même objet de désir mais seulement promesse d'une satisfaction à venir et dès longtemps préparée.

En apparence, c'est bien toujours le cas dans les trois fictions du siècle des Lumières. Mais la phase intermédiaire du couvent étant supprimée, la proximité physique avec l'enfant donne une coloration évidemment plus trouble à ces trois textes. Sans doute, la petite fille y est-elle bien encore perçue avant tout comme promesse de plaisirs. Ainsi, avant que la petite Céladine lui soit miraculeusement confiée par sa mère, Cornandouk examine attentivement « toutes les petites personnes qui [lui] paraiss[ent] promettre de la beauté » et jette d'abord son dévolu sur "une enfant de quatre ans qui annonçaient pour l'avenir des charmes piquants $\gg .{ }^{24}$ Et lorsque Borille forme le projet d'enlever Tiamy, la fille de ses voisins, un regard affûté a su déceler en elle une future beauté, alors qu'elle est à peine âgée de quinze mois («il crut remarquer qu'elle serait extrêmement jolie », MDA, t. 3, p. 137). L'œil de Liebman n'est pas moins perspicace : «la femme d'un de mes jardiniers vint à mettre au monde une fille qui me promettait l'assemblage des grâces. Aussitôt je saisis un projet, qui, à tout autre qu'à moi, eût paru extravagant » (Liebman, p. 123). Mais, comme le suggèrent les rêveries exaltées dans lesquelles se perdent les trois personnages, tout se passe comme si ces promesses de plaisirs qu'ils découvrent avec ravissement dans l'enfant élue étaient devenues en elles-mêmes sources de la plus grande jouissance. Car l'un des attraits essentiels de la petite fille à leurs yeux est que ce qui se donne à voir en elle laisse encore tout à désirer. C'est dans L'Emile (qui s'en étonnerait ?) qu'on trouve sans doute la théorie la plus fine de cette séduction, propre à l'enfance, de la promesse et de l'inachevé : de même, explique Rousseau, que le spectacle du printemps est plus agréable que celui de l'automne en ce qu'il incite l'imagination à «joindre celui des saisons qui doivent suivre » et à ajouter à "ces tendres bourgeons [...], les fleurs, les fruits, les ombrages, quelquefois les mystères qu'ils peuvent couvrir ", on trouve de même plus de charme à " contempler une belle enfance » que "la perfection de l'âge mûr ». Car dans l'un et l'autre cas, «l'imagination voit moins les objets comme ils seront que comme elle les désire, parce qu'il dépend d'elle de les choisir $\gg{ }^{25}$

C'est à la lumière de ces analyses que s'éclairent les rêveries délicieuses dans lesquelles s'abîment aussi bien Cornandouk, que Borille ou Liebman au spectacle de la petite fille, jeune pousse à peine germée qu'ils viennent de transplanter dans un enclos édénique conçu exprès pour elle. Délices de l'imagination, plus encore que du sentiment, dont

\footnotetext{
23 Dimension justement mise en lumière dans la mise en scène récente de Jacques Lassalle (Théâtre de l'Athénée, janvier 2001) qui évoquait, dans son programme, son intention « d'interroger l'arrière-pays vénéneux des comédies de Molière $»$.

24 Mouhy, Les Délices du sentiment (Paris, 1753), t. 2, p. 257 (désormais DS). Tous les italiques dans les citations sont nôtres.

${ }^{25}$ Rousseau, Emile ou de l'éducation (livre II), éd. T. L'Aminot, F. et P. Richard, Paris, Garnier, 1999, p. 175.
} 
Cornandouk fait lui-même l'aveu à Céladine, alors qu'elle est âgée de onze ans et qu'il a curieusement décidé de lui faire le récit détaillé de toute son entreprise :

Je méditais sur l'avenir heureux dont j'allais jouir. Céladine, ma petite Céladine deviendra grande et belle, j'aurais les prémices de son cœur, de sa raison et de ses charmes [...]. Céladine, tout enfant que vous étiez, je ne m'occupais dès ce moment que de vous, vous commenciez déjà mon bonheur et j'attendais patiemment que vous fussiez en âge de le consommer ( $D S$, t. 2, p. 268-269).

De même, «les apparences de beauté » que Borille remarque dans la petite Tiamy le charment et « le confirm[ent] dans sa résolution »: « il se promit de profiter de toutes les occasions d'embellir [sa] retraite. Il voulait y passer toute sa vie entre les bras d'une beauté qu'il aurait le plaisir de former à son gré, et d'élever pour lui seul » (MDA, t. 3, p. 146). Lui aussi s'apprête à attendre patiemment de « pouvoir cueillir une fleur [...] cultivée avec tant de soins et de peines » $(M D A$, t. 4, p. 14). Patience qui s'explique peut-être d'autant mieux que ce moment de rêverie est, en lui-même, source de félicité suprême. "Oh! quel plaisir je goûterai à l'entendre former ses premiers sons ! "s'exclame Liebman à propos d'Amélie : « mon nom sera la première parole qui lui échappera; ses premiers pas seront pour venir à moi ; ses premiers sentiments seront pour m'aimer » (Liebman, p. 124); et plus loin : « quelle volupté inconnue à tous les autres humains je me promettais » (p. 126).

De fait, durant toute la croissance de la jeune pousse, dont ces amants jardiniers observent avec émoi chaque étape, leurs espoirs ne sont guère déçus. La métaphore florale est particulièrement insistante dans Liebman: "à chaque instant, la beauté de cette aimable enfant me semblait croître et se développer. [...] Amélie entrait dans sa quatrième année ; j'admirais une rose même à son bouton; je suivais tous les degrés de croissance et d'embellissement » (Liebman, p. 125). Vient l'éclosion, qui comble tous ses vœux :

Mon Amélie avait atteint cet âge enchanteur... c'était alors la belle rose brillante de tout son éclat. [...] Chaque regard me causait un trouble que j'aimais à entretenir ; le beau front ! la candeur, la vérité, la nature même y respirait. Sa bouche, sa bouche était un bouton de rose dans toute sa rougeur virginale; c'était là que l'imagination enflammée se figurait la source de l'ambroisie et du nectar de la volupté... (Liebman, p. 127).

Métaphores nullement innocentes et qui ne sauraient manquer de faire surgir l'attente d'une défloration (le texte de La Solle est d'ailleurs, on l'a vu, explicite à ce sujet). Mais, dès cette phase a priori purement préparatoire, tout contact avec la petite fille n'est pas éludé et les rêveries voluptueuses s'alimentent aussi d'une proximité physique avec le corps de l'enfant. On relèvera en particulier une scène récurrente : celle de l'amant prenant la petite fille sur ses genoux, et manquant tomber en extase. Tel est le premier mouvement du prince Cornandouk, qui s'est interdit pourtant toute visite à la petite Céladine jusqu'à sa septième année (elle est jusque là élevée par une jeune africaine muette, sa seule compagne dans la tour où elle est enfermée), lorsqu'il s'introduit pour la première fois auprès d'elle : " la première chose qu'il fit [...] fut de me mettre sur ses genoux et de me considérer à la lueur d'une lanterne qu'il avait à la main [...]; il avait un air si content et si satisfait que tout ce que je pus comprendre c'est qu'il prenait un grand plaisir à me voir. Hélas ! je ne fus que trop confirmée dans cette opinion dans les suites.» (DS, t. 2, p. 237). De fait, Cornandouk prend vite l'habitude de lui apporter tous les jours de nouveaux jouets, de s'amuser avec elle durant de longues heures, non sans la reprendre sur ses genoux et « approch[er] sa bouche de la [s]ienne avec des ravissements » dont la petite fille ne saurait " comprendre la cause » $(D S, \mathrm{t} .2$, p. 239). 
Liebman est le seul à isoler l'enfant sans la séparer de sa mère. Moyen de garantir la pureté de ses intentions ${ }^{26}$ : seul un «engagement légitime» pourra lui procurer les plaisirs qu'il entend goûter avec elle (Liebman, p. 131). Mais en dépit de proclamations insistantes sur la noblesse et l'innocence de ses vœux, les émois de Liebman ne sont pas moins vifs que ceux de Cornandouk :

Ses caresses innocentes allumaient dans mon sein un feu qui chaque jour devenait plus dévorant ; combien de fois lui faisais-je répéter qu'elle m'aimait, qu'elle m'aimait de toute son âme ! [...] Quand je prenais cette enfant sur mes genoux, c'était alors qu'une langueur délicieuse coulait dans mes veines; je m'enivrais à longs traits de ma passion; je brûlais. Un doux frissonnement succédait à cette flamme rapide. (Liebman, p. 125-126).

Liebman a beau rassurer aussitôt son auditeur sur sa belle âme et sur la qualité de ses sentiments (« mais que les plaisirs de mon cœur était au-dessus de ceux de mes sens !»), c'est bien, semble-t-il, au fantasme d'une séduction entre adulte et enfant que l'on assiste ici. On relèvera d'ailleurs que c'est à la petite fille que sont attribuées les premières caresses qui «allument» le désir : « il fallait incessamment m'arracher aux caresses ingénues de cette divine enchanteresse; quel supplice ! c'était à moi, qui idolâtrais Amélie, à combattre, à repousser les témoignages naïfs de son amour?» (Liebman, p. 133).

Qu'on ait affaire ici à une scène topique et lourdement investie, c'est ce que suggère une séquence du récit de Bibiena intitulé La Poupée (1747). Le héros, un jeune abbé aveuglé par sa fatuité, tombe amoureux d'une poupée et découvre qu'il s'agit en réalité d'une belle sylphide, qui grandit à mesure qu'elle parvient à l'initier à une sagesse de l'amour :

Aussitôt que je vis ma poupée à cette taille où nous voyons ordinairement un enfant à peu près de cinq ans, ce feu voluptueux qui coulait dans mes veines se calma, je n'eus plus de désirs. [...] Je la baisai lorsque je l'eus mise sur mes genoux et qu'elle s'y fut arrangée avec cette facilité naturelle à un enfant qui ne connaît point de malice. [...] Cette petite gorge dont les charmes m'avaient transporté, enflammé il n'y avait qu'un moment, n'était plus pour moi qu'une gorge innocente qui ne m'inspirait aucun désir ni nulle envie de la toucher. Peu s'en fallut qu'il ne m'échappât plusieurs fois de l'appeler petite fille. ${ }^{27}$

Dénégations pour le moins insistantes et d'autant plus troublantes, comme l'a souligné Henri Lafon dans sa préface, que l'on sait que Bibiena sera accusé, en 1763, d'avoir violé la petite fille de ses logeurs, âgée de deux ans et neuf mois... Ces protestations d'innocence ont en tout cas le mérite de mettre en lumière la puissance de l'interdit qui borde les récits de Cornandouk et de Liebman. ${ }^{28}$

Borille n'est guère en reste. Avant d'enlever la petite Tiamy, il se concilie ses faveurs par ses caresses et «par des dragées et des colifichets» $(M D A$, t. 3, p. 137). Une fois sa

\footnotetext{
26 «J'avais rassuré les parents sur la suite du plan que je m'étais proposé ; mon dessein n'était pas d'abuser des mouvements favorables que je voulais exciter et échauffer. [...] Je n'eusse volé dans ses bras qu'appuyé des lois d'un saint engagement : mais je voulais m'assurer de son âme » (Liebman, p. 126).

27 Bibiena, La Poupée, éd. H. Lafon, Paris, Desjonquères, 1987, p. 111-112.

28 On retrouve d'ailleurs dans la nouvelle de Baculard d'Arnaud la même image pour évoquer le désir suscité par le corps de l'enfant placé sur les genoux, celle d'un feu brûlant qui «coule dans les veines » (élément simplement affecté d'un signe négatif dans le récit de Bibiena). Le héros de La Poupée tente d'expliquer cette conversion soudaine du désir en un attendrissement innocent : " aussitôt que j'ai vu [la sylphide] à une taille que nous connaissons et qui nous représente l'âge d'innocence, j'ai éprouvé cette pureté de sentiments que cet âge inspire » (La Poupée, p. 112). Explication qu'il ne tarde pas à juger lui-même peu « satisfaisante ». On est en droit de n'être guère plus satisfait du second argument, selon lequel la sylphide aurait elle-même suspendu en lui le désir pour mieux le faire renaître ensuite...
} 
« proie » en son pouvoir, il est surtout le seul des trois personnages à exclure toute présence d'un tiers. Ce qui l'oblige à prodiguer lui-même des soins ordinairement réservés à une mère, ou à une nourrice. Dès lors, l'intimité physique avec l'enfant ne saurait être que des plus étroites :

La petite, qui n'avait jamais reçu de lui que des caresses, ne fut point effrayée de se voir seule avec lui. Elle joua avec tout ce qui se présenta, et ses nouveaux amusements l'ayant bientôt fatiguée, il s'en aperçut, et après lui avoir fait manger un potage qu'il avait eu la précaution de faire porter dans sa chambre, il la déshabilla comme il avait souvent vu faire à sa mère, et la coucha dans le lit. Elle s'endormit bientôt [...]. Le lendemain matin il revint de bonne heure dans cette maison, et rendit à l'enfant tous les services que son âge pouvait exiger. (MDA, t. 3, p. 143-144)

Où l'on voit que cet isolement de la petite fille qu'on a désigné comme la troisième composante du topos de la "précaution inutile» fait l'objet, lui aussi, d'une singulière radicalisation par rapport aux fictions du XVIIe siècle. Différence de degré d'abord: l'entreprise de séquestration, déjà minutieusement agencée chez Cervantès et Molière, devient totalement frénétique chez Mouhy et La Solle. A peine la petite Céladine lui a-t-elle été confiée par sa mère que Cornandouk enferme son trésor dans un lieu secret, au fond d'une tour inexpugnable. Ce n'est pas assez pour rassurer ses craintes : à la suite d'un cauchemar (évidemment prophétique) qui lui montre les portes de la tour ouvertes et la petite fille disparue, Cornandouk imagine deux moyens de «rendre cette solitude inaccessible et impénétrable»: premièrement de «peupler son second fossé de bêtes féroces », deuxièmement de persuader le Roi son frère de sa mort afin d'éviter toute intrusion $(D S, \mathrm{t}$. 2 , p. 271-272). Pour être décrite par La Solle avec un plus grand souci de vraisemblance, la forteresse conçue par Borille n'en paraît pas moins impénétrable que celle de Cornandouk. La maison destinée à la claustration de Tiamy ainsi que les travaux permettant de rendre son existence absolument secrète font d'ailleurs l'objet de descriptions dont Henri Lafon a souligné l'« exceptionnelle minutie ». ${ }^{29}$

Plus fondamentalement, l'isolement de l'enfant change de nature dans ces trois fictions des Lumières, et devient indissociable de la dimension radicalement " négative » de l'éducation qu'elle reçoit. Car la réclusion de Céladine, Tiamy et Amélie est telle qu'elle aboutit à une complète néantisation du monde extérieur, dont elles ignorent jusqu'à l'existence. A cet égard, le projet de Cornandouk est sans ambiguïté : il s'agit «d'enlever une fille en si bas âge qu'elle ne put conserver aucune idée de ses parents, ni du monde dont [il] la sèvrerai $[\mathrm{t}] »$. $(D S$, t. 2 , p. 256). Au frère de Cornandouk, qui est parvenu à la délivrer, Céladine (alors âgée environ de douze ans) avoue d'ailleurs : «J'ai été heureuse [ici] tant que j'ai ignorée que j'y étais enfermée et qu'il y avait d'autres lieux étendus, éclairés par une lumière céleste et habités par d'autres êtres que par Cornandouk et par moi » $(D S, \mathrm{t}$. 2, p. 232). Ignorance et isolement plus complets encore pour Tiamy, élevée seule par Borille. Désireux de la distraire, et espérant aussi rendre sa compagnie plus amusante, il songe un instant à lui apprendre à lire. Mais il renonce vite à ce projet, qui pourrait l'éclairer sur tout ce qu'il s'acharne à lui dissimuler :

En lisant elle apprenait [sic] mille choses qu'il voulait lui laisser ignorer; elle saurait qu'il y avait d'autres hommes, et il ne voulait pas qu'elle le sut, persuadé qu'elle mettrait tout en usage pour parvenir à les connaître ; [...] elle saurait qu'il y a des pays, des villes, des maisons que le soleil éclaire, que la nuit couvre de ses voiles,

29 H. Lafon, Les Décors et les choses dans le roman français du XVIIIe siècle, Oxford, Voltaire Foundation, 1992, SVEC 297, p. 300. Voir aussi notre livre : Espaces du féminin dans le roman français du XVIIIe siècle, Oxford, Voltaire Foundation, SVEC 2004 : 01, p. 310-316. 
et il fallait qu'elle crut qu'elle existait seule avec son maître, et que son appartement était tout l'univers... (MDA, t. 4, p. 4).

A première vue, l'isolement d'Amélie peut sembler moins absolu: seule des trois petites filles à bénéficier de la présence de sa mère, elle est la seule aussi à jouir de la lumière du jour. Mais la « fort belle terre » où Liebman s'est « enseveli » (Liebman, p. 122) lui permet de maintenir sa proie dans une parfaite ignorance du monde qui l'entoure : «il est décidé qu'Amélie ne verra que sa mère et moi, que ses premiers regards ne se partageront qu'entre nous deux [...]. Je veux qu'elle imagine qu'il n'y a sur la terre, excepté elle et sa mère, d'autre créature que son amant» (p. 124). Lors des promenades, Liebman prend d'ailleurs « la précaution d'écarter les moindres témoins » (p. 125).

Si le vœu d'Arnolphe était de « choisir une moitié qui tienne tout de [lui] », ${ }^{30}$ celui de ses successeurs est de vivre en compagnie d'un être auquel ils puissent " tenir lieu de tout", pour reprendre une formule de Cornandouk. ${ }^{31}$ Ainsi de Liebman, dont le désir le plus cher est d'être "l'unique objet» des sentiments, des actions et des moindres pensées d'Amélie. ${ }^{32}$ Fantasme d'une emprise totale qui se complète d'une identification manifeste au Dieu créateur de la Genèse : dans les Mémoires de deux amis, Borille souhaite que Tiamy croie qu'il est la source de toute lumière. ${ }^{33}$ Et lorsque Amélie demande à Liebman « qui a fait ces jardins, ces gazons, ces fleurs, qui a distribué ces eaux étincelantes dans ces bassins...», ce dernier n'hésite pas à s'en attribuer la paternité : «Qui, mon Amélie ?... qui ?... ton ami, ton amant, moi ; le désir de te plaire m'a animé, et j'ai produit toutes ces merveilles » (Liebman, p. 129). Verbe qui ne peut être que fort équivoque aux oreilles de la petite fille, et qui atteste chez Liebman le désir d'être regardé par elle comme un Dieu. Mais les questions d'Amélie sont insistantes ( « tu as créé cette voûte arrondie où se peint un bleu qui charme ma vue, ce soleil qui me transporte de ravissement ?»), et Liebman doit reconnaître qu'il n'est point «l'auteur de ces miracles », finissant par introduire l'idée d'un Dieu créateur dans l'esprit de la fillette. Quant à Cornandouk, s'il manifeste davantage de scrupules que Liebman à s'attribuer des pouvoirs démiurgiques, il n'en est pas moins fort «embarrassé » d'avouer à la petite Céladine que ce n'est pas lui qui a fait les fleurs qu'il lui apporte et qu'elles sont un « ouvrage de la nature » $(D S$, t. 2, p. 288$)$.

$\mathrm{Au}$ reste, l'enclos dans lesquels s'enferment les trois nouveaux Pygmalions en compagnie de leur enfant-Galathée est visiblement conçu comme une recréation du jardin d'Eden, ainsi que l'atteste en particulier la nudité originelle que Borille impose à la petite Tiamy. ${ }^{34}$ Déjà la fascination d'Arnolphe pour la naïveté et la supposée «sottise » d'Agnès renvoyaient au fantasme d'une nature primitive, antérieure à toute corruption. C'est bien aussi comme un fragment de nature non dénaturée qu'est perçue la petite fille dans ces trois fictions des Lumières. La nature érotique de la manipulation dont ces fillettes sont l'objet incite certes leur amant à ne pas renoncer absolument aux agréments de l'art: le héros de La Solle enseigne ainsi à Tiamy les raffinements de la toilette. Mais il ne s'agit que de lui apprendre à

\footnotetext{
${ }^{30}$ L'Ecole des femmes, I, sc.1 (v. 126).

31 «Elle ne verra jamais que moi, elle m'aimera, je lui tiendrai lieu de tout et elle fera le comble de mes désirs » $(D S$, t. 2, p. 268).

32 « Il y aura donc dans l'univers, une créature qui n'existera que pour moi, qui ne sera remplie que du seul désir de me plaire, qui m'aimera sans partage! je serai l'unique objet auquel se rapporteront ses sentiments, ses actions, ses plus indifférentes pensées! »(Liebman, p. 126).

33 « Il fallait qu'elle crut [...] qu'il n'y avait point d'autre lumière que celle de sa lampe que son maître seul savait rendre perpétuel en y mettant de l'huile» (MDA, t. 4, p. 4).

34 «Comme il n'entrait point d'un air étranger [dans la chambre], il y faisait fort chaud, il voulait ainsi accoutumer sa belle à être toujours nue, et il y réussit, ayant l'attention de se déshabiller toutes les fois qu'il entrait chez elle. » (MDA, t. 3, p. 147). Participe aussi de ce code édénique la crainte que les livres, comme les fruits de l'arbre de la connaissance dans le récit de la Genèse, n' « ouvrent les yeux » à Tiamy.
} 
« relever ses charmes naturels par le secours d'un art innocent» $(M D A$, t. 4, p. 10). Amélie incarne ce même idéal aux yeux de Liebman : "c'était la nature dans ses attraits ingénus, parée des ornements de l'art » (Liebman, p. 166). Car l'attrait érotique le plus puissant de la petite fille, c'est bien cette ingénuité et cette innocence qu'il importe à tout prix de préserver, pour s'offrir le spectacle ravissant d'une humanité en son enfance: «je n'avais point le dessein d'abuser de la naïveté d'une créature enchanteresse qui n'avait nulle idée des convenances, qui ne sachant point ce que c'était que rougir, était, en un mot, dans une ignorance absolue du mal» (Liebman, p. 128). A bien des égards, le comportement de Céladine et de Tiamy n'est d'ailleurs pas sans faire songer à celui que Rousseau attribue au sauvage dans le Discours sur l'origine de l'inégalité en 1755. Céladine décrit ainsi son enfance auprès de l'esclave muette qui est sa seule compagne: « je vivais avec Malga, sans réflexion, sans désir et même sans penser » $(D S$, t. 2, p. 234). Quant à la petite Tiamy, « livrée machinalement au premier objet qui attachait ses regards, elle passait des jours tranquilles, parce qu'elle ne connaissait pas de conditions plus agréables que la sienne. [...] Elle ne connaissait pas d'autre plaisir que de satisfaire aux besoins de la nature » (MDA, t. 4, p. 6 et p. 8). Qu'on se rappelle le portrait du sauvage selon Rousseau : "ses désirs ne passent pas ses besoins physiques [...]. Son imagination ne lui peint rien ; son cœur ne lui demande rien [...] son âme que rien n'agite se livre au seul sentiment de son existence actuelle $» .^{35}$

Là est bien ce qui distingue le plus radicalement, sans doute, ces trois fictions de leurs modèles classiques : l'isolement de la petite fille semble destiné à combler non plus seulement une pulsion érotique mais bel et bien aussi une pulsion «épistémophilique ». Dans ces trois récits, affleure assez nettement, en effet, un fantasme expérimental d'observation d'un être neuf, méticuleusement soustrait au processus usuel d'éducation et de socialisation. Tout se passe en fait comme si ces fictions opéraient une sorte de contamination entre le motif de la «précaution inutile » et un motif au moins aussi ancien, puisqu'il remonte à Hérodote, mais qui s'épanouit dans la seconde moitié du XVIIIe siècle: celui d'une expérimentation sur l'origine. ${ }^{36}$ A cet égard, la petite Imirce, l'héroïne du roman éponyme de Du Laurens (1765), est assurément une proche cousine de Céladine, Tiamy et Amélie puisque, enfermée par un " philosophe » dans une cave " dès les premiers jours de sa naissance » en compagnie d'un garçon du même âge, son enfance dure vingt-deux ans. ${ }^{37}$ Dans son cas néanmoins, le rapport de subordination entre la visée expérimentale et la portée érotique de la séquestration semble inversé puisque c'est seulement après l'avoir observée durant de longues années qu'Ariste s'avise de sa beauté et la retire de sa prison. ${ }^{38}$ A l'inverse, la dimension expérimentale reste à l'arrière-plan des récits de Mouhy, La Solle et Baculard d'Arnaud. C'est bien elle pourtant qui donne à ces trois textes leur aspect si troublant.

\footnotetext{
35 Rousseau, Discours sur l'origine et les fondements de l'inégalité parmi les hommes, éd. J. Starobinski, Paris, Gallimard, 1969, 73-74. On sait que ce portrait est très proche de celui que Rousseau fait de l'enfant au livre II de l'Emile, «tout entier à son être actuel et jouissant d'une plénitude de vie qui semble vouloir s'étendre hors de lui » (éd. citée, p. 255).

36 Sur la postérité des expériences d'isolement enfantin (expérience de Psammétique) au siècle des Lumières, voir Jean-Michel Racault, « Le motif de l'enfant de la nature dans la littérature du XVIIIe siècle ou la recréation expérimentale de l'origine » in Primitivisme et mythe des origines dans la France des Lumières, 1680-1820, (Paris, 1989, p. 101-117) et Béatrice Durand-Sendrail, "L'Elève de la nature de Gaspard Guillard de Beaurieu : un avatar de l'expérience de Psammétique », Transactions of the ninth international congress on the Enlightenment (Münster 1995), SVEC 346-348, 1997, t. I, p. 381-384.

37 Tels sont les premiers mots de son récit: «Je suis née en France, je ne sais dans quelle province; je n’ai connu ni père, ni mère ; mon enfance a duré vingt-deux ans ; jusqu'à cet âge, je n'ai vu ni le ciel, ni la terre » (Du Laurens, Imirce ou la Fille de la nature, éd. A. Rivara, Presses de l’Université de Saint-Étienne, 1993, p. $71)$.

38 « J'étais depuis vingt-deux ans dans cette prison. [...] Ariste s'aperçut que j'étais jolie, me soupçonna de l'esprit, conçut de l'amour pour moi, et me tira de sa cave » (ibid., p. 75).
} 
Céladine souligne ainsi la "bizarrerie cruelle» de sa séquestration, qu' « aucun motif raisonnable » ne saurait justifier $(D S$, t. 3, p. 37). Moyen de suggérer que sa captivité renvoie à des motivations plus opaques que le dégoût du monde et la défiance envers les femmes invoqués par son geôlier. De fait, c'est à une interminable "épreuve » que Cornandouk soumet la petite fille. ${ }^{39}$ Même dans le langage purement sentimental de Liebman, qui dénie avec obstination toute tentation libertine dans son projet, surgit la notion d' «expérience » pour qualifier la pédagogie négative qu'il pratique avec Amélie: «je reculais toujours l'instant où l'honnêteté et le devoir m'ordonnaient de l'éclairer; sans doute cette sorte d'expérience offensait le ciel ». Phrase clef assurément puisque c'est à peu près la seule où Liebman ne se pose pas en victime du sort et assume partiellement la responsabilité du drame. A maintes reprises dans son récit, transparaît d'ailleurs une avidité à observer la petite fille, indissociablement perçue comme objet de désir et objet d'étude : "mes yeux ne se détachaient point d'Amélie ; j'épiais son premier regard, son premier sourire » (Liebman, p. 125). Mais c'est sans doute chez Borille que le désir d'expérimentation est le plus manifeste. En témoigne notamment le projet un instant caressé d'enseigner le dessin à Tiamy : « le défaut de connaissance du monde, et la vivacité naturelle pourraient faire de singuliers effets sur l'imagination de cette élève et produire des dessins dont la bizarrerie mériterait l'admiration des connaisseurs » $(M D A$, t. 4, p. 6). Tiamy est la seule d'ailleurs à ne pas être alphabétisée. Bien plus, elle n'accède pas même à l'usage de la parole : " Il ne lui apprit même pas à parler; seulement il lui nomma les choses de l'usage le plus commun, comme le pain, le vin, la viande, un lit, une chaise, et il fut content quand il la vit en état de lui dire qu'elle avait faim ou soif, froid ou chaud» $(M D A$, t. 4, p. 5). Il s'agit en réalité de maintenir la petite fille à ce stade tout à fait primitif de l'usage des signes que Condillac analysait dans son Essai sur l'origine des connaissances humaines : celui où l'homme ne connaît encore " d'autres mots que les noms [donnés] aux objets sensibles $\gg .{ }^{40}$

Cette dimension expérimentale dans l'isolement de la petite fille conduit à une subversion complète du schéma de la « précaution inutile », que l'on a pourtant désigné plus haut comme la donnée la plus essentielle du topos. Car loin de manifester la vanité dérisoire du projet de séquestration, le lent éveil au monde de la petite fille semble faire partie du processus même de l'expérimentation. Sans doute Cornandouk et Liebman opposent-ils de vives résistances au désir de savoir qui se manifeste peu à peu chez leur prisonnière. Mais à vrai dire, le dévoilement progressif de la vérité est aussi pour eux source de jouissances non négligeables. En témoigne la solennité avec laquelle Cornandouk décide d'ôter (partiellement) le voile si longtemps posé sur les yeux de Céladine : «écoutez-moi de toutes vos oreilles, je vais mettre fin à l'ignorance dans laquelle vous avez vécu jusqu'ici, et vous apprendre bien des vérités. Il y a un autre monde que celui où vous vivez depuis que vous êtes née ». Dans son propre récit rétrospectif, la petite fille suggère l'attention avec laquelle Cornandouk observe l'effet que ce dévoilement produit sur elle. ${ }^{41}$ On ne saurait non plus douter de la jouissance qu'éprouve Liebman à «lever le grand rideau qui cachait la vérité aux yeux d'Amélie » (Liebman, p. 134). Le héros de Baculard d'Arnaud organise d'ailleurs cette révélation comme un véritable spectacle : adoptant « avec transport» le plan que lui soumet son ami Rimberg, il décide de faire prendre à la petite fille « une liqueur soporative » destinée à l'assoupir quelques heures : «pendant ce temps, on la transporterait dans un endroit où pourrait se trouver une multitude d'hommes, comme au spectacle, dans une promenade, etc. J'épierais le premier moment de son réveil et je jouirais de toute sa surprise » (Liebman, p.

\footnotetext{
${ }^{39}$ Le mot est prononcé par Céladine à propos de la manière dont Cornandouk lui distille les informations sur sa captivité (voir $D S$, t. 2, p. 267).

40 Condillac, Essai sur l'origine des connaissances humaines, Amsterdam, 1746, t. 2, p. 119.

41 «Cornandouk [s'arrêta] dans cet endroit de son histoire, sans doute pour observer l'effet d'un récit qui devait naturellement me remuer » $(D S$, t. 2 , p. 267).
} 
135). Expérience clairement libertine en son principe, source d'une jouissance tout à fait comparable à celle que se procurait le Prince philosophe de La Dispute de Marivaux (1744) en observant l'étonnement et le trouble de créatures ingénues découvrant le monde après avoir été isolés jusqu'à l'adolescence : "vous devez vous figurer, monsieur, la singularité de cette situation, et la source des impressions toutes neuves qui en résultèrent » (Liebman, p. 138).

Si Borille se prive d'une situation aussi « singulière » et des jouissances qu'elle procure, il n'en va pas de même de son neveu, Rinville, qui hérite à sa mort de sa maison et découvre la cachette de Tiamy (devenue une belle jeune femme), et de ses quatre enfants. Loin d'apparaître comme un libérateur, Rinville semble prendre le relais du fantasme expérimental de son oncle : son premier geste consiste à introduire de la nourriture dans leur chambre et à observer avec la curiosité d'un naturaliste le repas de « cette famille de sauvages » (MDA, t. 4, p. 95). Et si, dès le lendemain, il laisse Tiamy sortir de la chambre, c'est moins, semble-t-il, pour la délivrer que pour jouir du spectacle de son étonnement devant l'éclat du soleil, et l'aspect des fleurs et des arbres d'un jardin. ${ }^{42}$

On voit que la contamination du scénario de la "précaution inutile» et du motif de l'isolement expérimental n'est pas sans effet : à la phase de séquestration ne succède plus une véritable libération mais une phase d'acculturation qui a pour objet l'observation des réactions de la petite fille devant le monde qu'on lui dévoile peu à peu. Même dans l'" Histoire de Céladine », récit dont la logique narrative reste la plus conforme au schéma traditionnel, la libération de la belle captive par le frère de Cornandouk est précédée (dans la chronologie interne du récit et non dans le temps de la narration) par une longue phase durant laquelle les « idées » de Céladine se « développent » au fur et à mesure des révélations parcimonieuses de son geôlier $(D S$, t. 2, p. 287).

Au reste, loin d'être inutiles, les "précautions » d'un Borille ou d'un Liebman sont d'une efficacité remarquable. Jusqu'à la mort, tout à fait naturelle, du premier, rien ne vient perturber ses plans : Tiamy est bel et bien façonnée en une sorte de poupée vivante, seulement désireuse d'assouvir les désirs de celui qu'elle désigne du nom de « Maître ». La réussite de Borille n'est certes pas totale, puisque la jeune fille n'est pas absolument abêtie : « La raison de Tiamy, par le secours des années, perçait à travers les ténèbres épaisses dont l'ignorance la couvrait » $(M D A$, t. 4, p. 12). Mais rien néanmoins ne vient troubler des plaisirs auxquels Tiamy se livre « sans art » et Borille « sans réserve » (MDA, t. 4, p. 17). Quant à Liebman, son vœu le plus cher est parfaitement exaucé : lui qui aurait « voulu trouver un cœur qui n'eût respiré que par le [s]ien », et dont le dessein était de faire élever une petite fille "pour en créer l'objet d'un amour » qu'il serait le seul capable de ressentir (Liebman, p. 122 et p. 125), trouve (ou fabrique) en Amélie une créature qui répond parfaitement à son attente, comme elle ne cesse de le lui répéter : "Tu demandes toujours, Liebman, si je t'aime? me serait-il possible d'avoir un autre sentiment? un mouvement dont $\mathrm{j}$ 'ignore la cause me porte dans tes bras, m'y précipite $» .{ }^{43}$ Et si le personnage de Rimberg joue un rôle non négligeable dans la catastrophe finale, ce n'est nullement à la manière d'un Horace contrariant les plans d'Arnolphe. C'est Liebman lui-même qui a introduit cet ami de jeunesse dans son enclos

\footnotetext{
42 «Les arbres du jardin, le parterre, chaque fleur en particulier l'occupait d'une manière ravissante. Le hasard avait interrompu mon sommeil, le plaisir de jouir de la surprise de Tiamy le suspendit entièrement. » (MDA, t. 4, p. 109). Le même soir, Rinville succombe d'ailleurs à « une faiblesse criminelle » avec Tiamy, ce qui semble bien faire de lui une sorte de double de son oncle. On soulignera au passage que, sans lui être assimilable, le personnage d'Horace, dans la comédie de Molière, prenait bien en un sens le relais d'Arnolphe : "l'heureux naturel », la « tendresse innocente » et « l'ingénuité » qu'il apprécie en la jeune Agnès (voir L'Ecole des femmes, III, sc.4, v. 943-945) ne sont-ils pas, au moins partiellement, les fruits du patient «travail » d'Arnolphe et de l'éducation négative imposée à la petite fille?

${ }^{43}$ Liebman, p. 128. Liebman ne doutait d'ailleurs pas un instant du succès de son projet au moment même de le concevoir : « ses premiers sentiments seront pour m'aimer ; et comment ne lui serais-je pas cher? je volerai audevant de ses moindres désirs » (p. 124).
} 
paradisiaque, pour des motifs on ne peut plus troubles : l'envie qu'il suscite chez Rimberg en lui offrant secrètement le spectacle de la petite Amélie semble surtout pour le héros le moyen de mieux «sentir la valeur» de son trésor (Liebman, p. 134). Rival d'autant plus fantasmatique donc, que Rimberg ne parvient nullement à séduire la jeune fille (laquelle n'a littéralement d'yeux que pour Liebman), mais seulement à la persuader qu'elle est trahie pour une autre femme, la baronne Dénof. Tel est sans doute le seul élément (lointainement) hérité de la tradition de la "précaution inutile » : l'ironie (à valeur tragique en l'occurrence) du drame de Liebman tient au fait que la naïveté si patiemment entretenue de la petite fille est l'une des causes du désastre. ${ }^{44}$ Plus fondamentalement, le succès même de l'entreprise conduit à la catastrophe : conformément aux vœux de Liebman, Amélie ne "respire » bel et bien que par lui, et c'est la jalousie et le narcissisme de son Pygmalion qui entraîne son suicide (par empoisonnement...).

A la différence de Carrizalès ou Arnolphe, Borille et Liebman parviennent donc à « exciter et échauffer» des "mouvements favorables» chez leur petite prisonnière. ${ }^{45}$ Et si Cornandouk échoue finalement à en faire autant auprès de Céladine, tout à coup " refroidie » par le récit de sa séquestration $(D S$, t. 2, p. 285), il n'en réussit pas moins d'abord à susciter chez elle un vif attachement : "j'étais si bien accoutumé à vivre avec lui, que j'étais triste quand il me quittait, et qu'aussitôt qu'il reparaissait, je reprenais ma joie » (DS, t. 2, p. 237). Cornandouk n'est même pas loin de se persuader, devant le silence de la petite fille, qu'il a réussi à étouffer en elle " la voix de la nature et de la liberté » $(D S$, t. 2, p. 266). C'est dire que, dans au moins deux des trois textes considérés, le vœu chimérique d'Arnolphe de " [se] faire une femme au gré de [son] souhait» (L'Ecole des femmes, I, sc.1, v. 142), et son fantasme démiurgique ("Comme un morceau de cire entre mes mains elle est, / Et je lui puis donner la forme qui me plaît», III, sc. 3, v. 810-811) sont quasiment devenus " réalités ». Sans doute les désirs de Cornandouk ni ceux de Liebman ne sont-ils parfaitement accomplis, puisque ni l'un ni l'autre ne jouissent de leur proie. Mais tout se passe comme si l'obstacle avait été chez eux intériorisé. ${ }^{46}$ C'est sans doute, en effet, en termes de pulsion d'échec, ou de névrose de destinée qu'il conviendrait d'appréhender ce qui fait obstacle à l'accomplissement de leur désir manifeste : en dévoilant tout son stratagème à la petite Céladine, Cornandouk suscite en elle des résistances insurmontables; et c'est au moment précis où Liebman s'apprête à épouser Amélie qu'il introduit Rimberg en tiers, déclenchant la catastrophe et produisant l'inaccessibilité de celle dont la possession était pourtant censée combler tous ses désirs. Au XVIIIe siècle, on le voit, les fantasmes pygmalionistes continuent de se heurter à de puissants interdits (dans le récit de La Solle, on en verrait volontiers le signe déplacé dans la peur maladive de la mort qui affecte Borille : "l'angoisse de la mort », n'est-elle pas « issue le plus souvent d'une conscience de culpabilité » $?^{47}$ ).

Reste à s'interroger, pour finir, sur les raisons qui ont pu conduire, au siècle des Lumières, à la « réalisation » fictionnelle de ce qui jusqu'alors ne pouvait être présenté que

\footnotetext{
44 On songe notamment à l'ironie (à valeur comique) du dénouement dans La Précaution inutile de Scarron : la jeune Laure trompe la confiance du vieux barbon en raison même de sa sottise et de sa naïveté et sans s'apercevoir qu'elle le trahit. En réalité, la séquence de Liebman est sans doute inspirée par un épisode fameux du Cleveland de Prévost, lorsque la naïve Fanny est persuadée par Gélin que Cleveland la trahit au profit de Mme Lallin.

45 Pour reprendre les termes suggestifs de Liebman (Liebman, p. 126).

46 On sait que, dans la première moitié du XVIIIe siècle, cette intériorisation de l'obstacle fut aussi l'innovation majeure de la dramaturgie marivaudienne par rapport au modèle moliéresque (voir Frédéric Deloffre, Une préciosité nouvelle : Marivaux et le marivaudage, Paris, Les Belles Lettres, 1955).

47 Freud, « Notre relation à la mort », in Essais de psychanalyse, Paris, Gallimard, 1981, p. 36.
} 
comme des projets chimériques et vains. ${ }^{48}$ Comment expliquer que le fantasme d'une emprise totale sur l'enfant, d'un modelage de son être, ne soit plus marqué du sceau du ridicule ou de la dérision? En fait, l'accomplissement fictionnel de ce rêve de toute-puissance renvoie sans doute à un changement d'epistémè. Avec l'avènement de l'empirisme, en effet, l'idée d'une nature humaine parfaitement malléable n'est plus inimaginable. D'où, au XVIIIe siècle, le développement d'un véritable «mythe de l'éducation ».49 Roger Mercier l'a montré : dans la philosophie des Lumières, « en face de [la] toute-puissance de l'éducation, la part du naturel est très réduite dans le caractère humain $\gg .^{50}$ Par le postulat d'une malléabilité presque parfaite de l'être humain, l'empirisme offre sans doute à Borille ou Liebman l'anthropologie qui manquait à Arnolphe et Carrizalès. D'autant que, dans l'imaginaire du XVIIIe siècle, cette malléabilité n'atteint pas le même degré chez tous les êtres : en raison de la mollesse et de la flexibilité de leurs « fibres », les femmes sont particulièrement propres à ce modelage. ${ }^{51}$ Mais les enfants le sont davantage encore : « les fibres [de l'enfant], molles et flexibles, prennent sans effort le pli qu'on leur donne; celles de l'homme, plus endurcies, ne changent plus qu'avec violence le pli qu'elles ont reçu ${ }^{52}$ On conçoit dès lors que, de cette fantasmatique propre aux Lumières, les petites filles aient été les objets d'élection.

Christophe Martin Université Michel de Montaigne - Bordeaux 3

\footnotetext{
48 Dans sa préface, La Solle revendique précisément le droit d'accomplir ce fantasme dans l'univers fictionnel : «pourquoi ne me serait-il pas permis de réaliser dans un roman des idées qui sont dans l'esprit de beaucoup de mes lecteurs?» $(M D A$, t. 1, p. IX).

49 Voir Jean Ehrard : « Naissance d'un mythe : l'éducation », in L'Idée de nature en France dans la première moitié du XVIIIe siècle, Paris, Albin Michel, 1994, p. 761-762.

${ }^{50}$ Roger Mercier, L'Enfant dans la société du XVIIIe siècle (avant l'Emile), Paris, 1961, p. 93.

51 En témoignent notamment ces considérations de Montesquieu : « les femmes ont les fibres plus molles, plus flexibles, plus délicates que les hommes » (Essai sur les causes qui peuvent affecter l'esprit et les caractères, in Euvres complètes, Paris, Seuil, 1964, p. 488).

52 Rousseau, Emile, éd. citée, p. 20.
} 